\title{
Lower diastolic wall strain is associated with coronary revascularization in patients with stable angina
}

Jaehuk Choi ${ }^{1}$, Min-Kyung Kang ${ }^{2 *}$ (D, Chaehoon Han², Sang Muk Hwang ${ }^{2}$, Sung Gu Jung ${ }^{2}$, Han-Kyul Kim², Kwang Jin Chun ${ }^{2,3}$, Seonghoon Choi ${ }^{2}$, Jung Rae $\mathrm{Cho}^{2}$ and Namho Lee ${ }^{2}$

\begin{abstract}
Background: Left ventricular (LV) diastolic dysfunction occurs earlier in the ischemic cascade than LV systolic dysfunction and electrocardiographic changes. Diastolic wall strain (DWS) has been proposed as a marker of LV diastolic stiffness. Therefore, the objectives of this study were to define the relationship between DWS and coronary revascularization and to evaluate other echocardiographic parameters in patients with stable angina who were undergoing coronary angiography (CAG).

Methods: Four hundred forty patients [mean age: $61 \pm 10 ; 249$ (57\%) men] undergoing CAG and with normal left ventricular systolic function without regional wall motion abnormalities were enrolled. Among them, 128 (29\%) patients underwent revascularization (percutaneous intervention: 117, bypass surgery: 11). All patients underwent echocardiography before CAG and the DWS was defined using posterior wall thickness (PWT) measurements from standard echocardiographic images [DWS = PWT(systole)-PWT(diastole)/PWT(systole)].

Results: Patients who underwent revascularization had a significantly lower DWS than those who did not $(0.26 \pm 0.08$ vs. $0.38 \pm 0.09, p<0.001)$. Age was comparable between the two groups (61 \pm 9 vs. $60 \pm 11, p=0.337)$, but the proportion of males was significantly higher among patients who underwent revascularization (69 vs. 52\%, $p=0.001)$. The LV ejection fraction was similar but slightly decreased $(60.9 \pm 5.7 \mathrm{vs.} 62.4 \pm 6.2 \%, p=0.019)$ and the E/E' ratio was elevated $(10.3 \pm 4.0$ vs. $9.0 \pm 3.1, p<0.001)$ among patients who underwent revascularization. In multiple regression analysis, lower DWS was an independent predictor of revascularization (cut-off value: 0.34; sensitivity: 89\%; AUC: 0.870; SE: 0.025; $p<0.001$ ).
\end{abstract}

Conclusion: DWS, a simple parameter that can be calculated from routine 2D echocardiography, is inversely associated with the presence of coronary artery disease and the need for revascularization.

Keywords: Echocardiography, Diastolic wall strain, Coronary revascularization

\section{Background}

Conditions causing chest pain or discomfort, such as an acute coronary syndrome or angina, have a potentially poor prognosis, emphasizing the importance of a prompt and accurate diagnosis [1]. For patients with acute coronary syndrome (ACS), 12-lead-electrocardiography (ECG), cardiac biomarkers, and echocardiography are used to confirm the diagnosis in combination with the characteristics

\footnotetext{
* Correspondence: homes78@naver.com

${ }^{2}$ Cardiology Division, Kangnam Sacred Heart Hospital, Hallym University

Medical Center, Seoul, South Korea

Full list of author information is available at the end of the article
}

of chest pain and conventional risk factors [1, 2]. Echocardiography is a good imaging modality for detecting a new loss of viable myocardium or new regional wall motion abnormality (RWMA) [1]. However, stable angina cannot be diagnosed or excluded by clinical assessment alone, and non-invasive functional imaging for myocardial ischemia, coronary computed tomography $(\mathrm{CT})$ calcium scanning or angiography, and coronary angiography (CAG) are often needed. Non-invasive functional imaging for myocardial ischemia uses myocardial perfusion scintigraphy with single-photon emission computed tomography (MPS with SPECT), stress echocardiography, first-pass 
contrast-enhanced magnetic resonance (MR) perfusion or MR imaging for stress-induced wall motion abnormalities [3]. Using research by Genders et al. [4], the ESC 2013 guideline contains a similar table of estimated risk percentages to the NICE 2010 guideline. Those with an estimated risk of $<15 \%$ should be presumed to not have coronary artery disease (CAD). If the estimated risk is $>15 \%$, the patient's left ventricular ejection fraction (LV EF) is a key determinant factor. Therefore, cardiac imaging plays a pivotal role in this type of decision- decision-making through determination of the LV systolic function and subsequent selection of a relevant intervention. However, EF cannot be used as an independent parameter to estimate the probability of the presence of CAD, but it can be used to help choose further studies to detect CAD in combination with clinical assessments. Therefore, routine 2-D echocardiography is less useful in patients with stable angina than in patients with ACS.

An echocardiographic study published in 1972 showed abnormal motion of the LV posterior wall during angina pectoris [5]. Recently, a non-invasive, load-independent, and reproducible estimator of LV stiffness using 2dimensional (2D) echocardiography, namely, diastolic wall strain (DWS), has been used to assess wall distensibility in the absence of LV systolic dysfunction [6]. Decreased DWS is associated with poor prognosis in patients with heart failure with preserved LV ejection fraction (HFpEF) [7] as well as in those with HF with reduced LVEF (HFrEF) [8] and is associated with adverse LV remodelling even in patients with normal LV systolic and diastolic function [9]. Therefore, the aims of this study were to evaluate the relationship between DWS and coronary revascularization as well as to evaluate echocardiographic and other parameters in patients with stable angina who underwent CAG.

\section{Methods}

\section{Study design and participants}

Of 2375 patients who underwent CAG from January 2013 to December 2015, 440 patients (57\% male, average age $=60$ years) who were diagnosed with or suspected to have stable angina were studied retrospectively. Patients were referred for evaluation of chest pain or dyspnoea by their general practitioner or by themselves. The character of the chest pain (typical, atypical, or nonanginal) was evaluated, and we excluded any patient with resting pain or suspected unstable angina. A variety of risk factors were recorded during the clinical assessment. Patients who had a prior history of CAD were excluded from this study regardless of their history of coronary stenting. All patients underwent CAG and pre-procedural EF by transthoracic echocardiography (TTE) and were sorted into two groups - the revascularization group $(n=128)$ and no revascularization group $(n=312)$. Patients with ACS, chronic kidney diseases, $\mathrm{LV} \mathrm{EF}<50 \%$, regional wall motion abnormalities, arrhythmia, severe valvular heart diseases, pericardial diseases, thyroid disease, moderate to severe pulmonary hypertension, sepsis, or haemodynamic instability were excluded from this study. We also collected participant data regarding demographic, anthropometric, and laboratory parameters including cardiac biomarkers.

\section{Echocardiography}

TTE was performed using standard techniques with a 2.5-MHz transducer. Standard 2-D and Doppler echocardiography was performed using a commercially available echocardiographic machine (Vivid 7R GE Medical System, Horten, Norway) with the same setup interfaced and a $2.5-\mathrm{MHz}$ phased-array probe. With the study participants in the partial left decubitus position and breathing normally, the observer obtained images from the parasternal long and short axes as well as from the apical four chamber and two-chamber and long-axis views. The depth setting was optimized to display the LV on the screen as large as possible and the same field depth was kept for both the four and two-chamber apical views. The sector width was reduced to increase the spatial and temporal resolution. The LV end-diastolic dimensions (LV EDD), end-diastolic interventricular septal thickness (IVSTd), and end-diastolic LV posterior wall thickness (PWTd) were measured at end-diastole according to the standards established by the American Society of Echocardiography [10]. LV EF was determined by the biplane Simpson's method. The maximal left atrial (LA) volume was calculated using the Simpson method and indexed to the body surface area. The LV mass was calculated using the Devereux formula: LV mass $=1.04$ [(LVEDD + IVSTd + PWTd $\left.)^{3}-(\text { LVEDD })^{3}\right]-13.6$. Thereafter, the LV mass index (LVMI) was calculated and indexed to the body surface area. DWS was calculated as [(PWTs) - (PWTd)/ (PWTs)] using M-mode echocardiography [6, 7].

The mitral flow velocities were recorded in the apical four-chamber view. The mitral inflow measurements included the peak early (E) and peak late flow velocities (A) as well as the E/A ratio. The tissue Doppler of the mitral annulus movement was also obtained from the apical four-chamber view. A 1.5-mm sample volume was placed sequentially at the septal annular sites. An analysis was performed for the early diastolic $\left(E^{\prime}\right)$ and late diastolic $\left(A^{\prime}\right)$ peak tissue velocities. As a noninvasive parameter for LV stiffness, the LV filling index (E/E') was calculated by the ratio of the transmitral flow velocity to the annular velocity. Adequate mitral and tissue Doppler image (TDI) signals were recorded in all patients.

The mean longitudinal global strain (GS) of LV was calculated from the apical 4,3,2-chamber views by speckle-tracking 2D-strain imaging [11]. 


\section{Carotid ultrasound}

A high-resolution B-mode ultrasound (Vivid 7R GE Medical Systems, Horten, Norway) equipped with a $7.5-\mathrm{MHz}$ linear array transducer was used for carotid ultrasonography. In the longitudinal view, the carotid intima-media thickness (IMT) was determined as the distance from the media adventitia interface to the intima lumen interface on the far wall in a region free of plaque [12]. The examiner assessed the presence of carotid plaques, which were defined as focal structures that encroached into the lumen by at least $100 \%$ of the surrounding IMT value. The common carotid artery IMT (CCA-IMT) was measured between the origin of the carotid bulb and a point $10 \mathrm{~mm}$ proximal to the CCA, and the carotid bulb IMT (CBIMT) was measured in the carotid bulb region. The CCAIMT and CB-IMT values were determined as the average of the maximum IMT of the left and right CCA and CB.

\section{Statistical analysis}

All of the continuous data are expressed as the mean \pm SD, and all of the categorical data are presented as percentages or absolute numbers. Continuous variables were analysed using Student's t-test, and dichotomous variables were analysed using the chi-square test. In addition, multivariate analysis (logistic regression, SPSS for Macintosh, version 10.0.7a, SPSS, Inc., Chicago, Ill., USA) was performed. All variables that had a $p$ value of 0.05 or less were considered statistically significant. The performance of continuous tests was assessed by receiver-operating-characteristic (ROC) curve.

\section{Results}

\section{Clinical parameters of the study population}

The clinical characteristics of the patients are shown in Table 1 . The study population included 440 patients who underwent CAG (mean age $60 \pm 10$ years) and 128 patients who underwent coronary revascularization. Patients in the revascularization group were predominantly male $(69 \%$ vs. $52 \%)$, and diabetes $(27 \%$ vs. $17 \%)$ and current smoker status (31\% vs. $22 \%)$ were more common in these patients. The incidence of coronary revascularization in men was 35\% (87/248) and in women was $21 \%$ (40/192), which was statistically significantly higher in males $(p=0.001)$.

\section{Echocardiographic parameters of the study population}

One hundred twenty-eight patients underwent coronary revascularization (percutaneous intervention: 113; bypass surgery: 14) among the 440 patients who underwent CAG. Echocardiographic measurements of the study population are shown in Table 2 . The entire study population had a normal cardiac size and systolic function, but

Table 1 Clinical parameters of the study population

\begin{tabular}{|c|c|c|c|}
\hline & Revascularization $(n=128)$ & No $(n=312)$ & $p$ \\
\hline Age (years) & $61.1 \pm 9.4$ & $60.0 \pm 10.5$ & 0.337 \\
\hline Male gender & $88(69 \%)$ & $161(52 \%)$ & 0.001 \\
\hline Systolic blood pressure $(\mathrm{mmHg})$ & $123.6 \pm 14.2$ & $121.1 \pm 15.4$ & 0.126 \\
\hline Diastolic blood pressure & $74.4 \pm 9.5$ & $73.5 \pm 9.9$ & 0.385 \\
\hline Heart rate (bpm) & $66.7 \pm 11.2$ & $66.4 \pm 12.4$ & 0.809 \\
\hline Body mass index $\left(\mathrm{kg} / \mathrm{m}^{2}\right)$ & $24.8 \pm 3.4$ & $25.2 \pm 3.2$ & 0.226 \\
\hline Hypertension & $69(54 \%)$ & $140(45 \%)$ & 0.093 \\
\hline Diabetes & $34(27 \%)$ & $54(17 \%)$ & 0.035 \\
\hline Stroke & $5(4 \%)$ & $16(5 \%)$ & 0.806 \\
\hline Current smoker & $40(31 \%)$ & $68(22 \%)$ & 0.051 \\
\hline \multicolumn{4}{|l|}{ Medications } \\
\hline RASB & $38(30 \%)$ & $82(27 \%)$ & 0.410 \\
\hline$C C B$ & $31(25 \%)$ & 74 (24\%) & 0.902 \\
\hline Statin & $40(32 \%)$ & $84(27 \%)$ & 0.350 \\
\hline Aspirin & $36(29 \%)$ & $67(21 \%)$ & 0.135 \\
\hline Clopidogrel & $10(7 \%)$ & $14(5 \%)$ & 0.167 \\
\hline Diuretics & $9(7 \%)$ & $28(9 \%)$ & 0.704 \\
\hline Beta-blocker & 17 (14\%) & 32 (10\%) & 0.402 \\
\hline Trimetazidine & $14(11 \%)$ & $19(6 \%)$ & 0.075 \\
\hline
\end{tabular}

Data are mean \pm standard deviation (SD) or or $\mathrm{n}(\%)$

CHD coronary heart disease, RASB Renin-Angiotensin system blocker, $C C B$ calcium-channel blocker 
Table 2 Echocardiographic parameters of the study population

\begin{tabular}{|c|c|c|c|}
\hline & Revascularization $(n=128)$ & No $(n=312)$ & $p$ \\
\hline$\overline{L A V I}\left(\mathrm{ml} / \mathrm{m}^{2}\right)$ & $22.7 \pm 6.9$ & $22.9 \pm 8.1$ & 0.862 \\
\hline $\operatorname{LVMI}\left(\mathrm{g} / \mathrm{m}^{2}\right)$ & $94.6 \pm 18.9$ & $91.1 \pm 22.2$ & 0.119 \\
\hline LV SWTd (mm) & $9.1 \pm 1.4$ & $8.9 \pm 1.4$ & 0.147 \\
\hline LV SWTS & $13.4 \pm 1.7$ & $13.0 \pm 1.8$ & 0.033 \\
\hline LV PWTd & $9.0 \pm 1.3$ & $8.7 \pm 1.3$ & 0.045 \\
\hline LV PWTS & $12.6 \pm 1.4$ & $13.9 \pm 1.6$ & $<0.001$ \\
\hline DWS & $0.26 \pm 0.08$ & $0.38 \pm 0.09$ & $<0.001$ \\
\hline LV EDD $(\mathrm{mm})$ & $49.9 \pm 3.5$ & $49.7 \pm 4.1$ & 0.632 \\
\hline LV ESD & $32.4 \pm 2.9$ & $31.7 \pm 3.6$ & 0.049 \\
\hline LV EF (\%) & $60.9 \pm 5.6$ & $62.4 \pm 6.2$ & 0.019 \\
\hline GS (\%) & $-17.4 \pm 2.5$ & $-18.3 \pm 2.9$ & 0.016 \\
\hline$E(\mathrm{~cm} / \mathrm{s})$ & $63.6 \pm 15.7$ & $63.8 \pm 15.6$ & 0.901 \\
\hline $\mathrm{A}(\mathrm{cm} / \mathrm{s})$ & $74.6 \pm 21.2$ & $71.5 \pm 17.8$ & 0.122 \\
\hline E/A ratio & $0.90 \pm 0.31$ & $0.93 \pm 0.32$ & 0.369 \\
\hline DT (ms) & $208.6 \pm 44.7$ & $202.0 \pm 44.2$ & 0.162 \\
\hline $\mathrm{E}^{\prime}(\mathrm{cm} / \mathrm{s})$ & $6.7 \pm 2.1$ & $7.6 \pm 2.5$ & 0.001 \\
\hline $\mathrm{A}^{\prime}(\mathrm{cm} / \mathrm{s})$ & $9.3 \pm 1.8$ & $9.3 \pm 1.8$ & 0.970 \\
\hline$E^{\prime} / A^{\prime}$ & $0.75 \pm 0.29$ & $0.85 \pm 0.34$ & 0.005 \\
\hline$E / E^{\prime}$ & $10.3 \pm 4.0$ & $9.1 \pm 3.1$ & $<0.001$ \\
\hline $\mathrm{S}^{\prime}(\mathrm{cm} / \mathrm{s})$ & $7.4 \pm 1.5$ & $7.6 \pm 1.6$ & 0.221 \\
\hline Diastolic grade & & & $<0.001$ \\
\hline normal & $22(19 \%)$ & $94(31 \%)$ & \\
\hline Grade 1 & $83(70 \%)$ & $201(66 \%)$ & \\
\hline Grade 2 & $14(11 \%)$ & $9(3 \%)$ & \\
\hline
\end{tabular}

Data are represented as mean \pm SD or $n(\%)$

LAVI left atrial volume index, $L V M I$ left ventricular mass index, LVEDD and ESD LV end-diastolic and systolic dimension, EF ejection fraction, GS global strain, $D T$ deceleration time

patients in the revascularization group had a slightly but significantly decreased LV systolic end-dimension, EF, and GS. Patients in the revascularization group also had a lower LV posterior wall thickness during systole and DWS. Figure 1 shows normal (a) vs. decreased DWS (b). Patients who underwent revascularization had a more advanced diastolic dysfunction and poorer diastolic parameters (lower E' velocity and elevated E/E'). In the analysis according to gender, \pm 0.09 vs. $8 \pm 0.10, p=0.001$ ). However, the mean DWS was significantly lower in the coronary revascularization group in both men $(0.38 \pm 0.08$ vs. $0.28 \pm 0.07, p<0.001)$ and women $(0.42 \pm 0.08$ vs. $0.29 \pm$ $0.10, p<0.001)$.

\section{Laboratory findings and other parameters}

Table 3 shows the laboratory parameters and results from the carotid ultrasound. Serum creatinine was higher in patients in the revascularization group. The mean IMT and maximal plaque thickness were higher in patients in the revascularization group.
The angiography results of 127 patients who underwent coronary revascularization

Table 4 shows the angiography results of 127 patients who underwent coronary revascularization. Fifty-six patients had single vessel disease (VD), and 71 patients had multivessel involvement. Most patients underwent one stent implantation, and one or more stent implantations were performed in 32 patients. The left anterior descending artery (LAD) was the most common target lesion, and $26 \%$ of patients had multiple lesions. All patients underwent coronary revascularization-confirmed complete revascularization (CR). CR was defined as a final angiography result without coronary stenosis $\geq 70 \%$ in major epicardial vessels or stenosis $\geq 50 \%$ in the left main [13].

\section{Univariate analysis}

Univariate analysis showed that several clinical, echocardiographic, and other factors had predictive value for coronary revascularization. A lower DWS, decreased GS, diastolic dysfunction, elevated E' velocity, male gender, diabetes, current smoking, higher IMT and higher maximal plaque thickness were associated with coronary revascularization in patients with clinical suspicion of stable angina in those who underwent CAG (Table 5).

\section{Multivariate analyses}

Among the variables found to be correlated with coronary revascularization, a lower DWS (OR: 0.920, CI: $0.862-0.981, p=0.011$ ) and higher IMT (OR: 12.629, CI: 1.277-124.817, $p=0.030$ ) were found to be independently related to coronary revascularization (Table 5). To better analyse the predictive value of the relationship between DWS and coronary revascularization, we used ROC curve analysis to determine if any threshold value of DWS existed. Inspection of the ROC curve showed that DWS $<0.34$ was associated with the need for coronary revascularization (sensitivity of $89 \%$ and specificity of 48\%, AUC: 0.870 , SE: 0.025, $p<0.001$, Fig. $2 \mathrm{a}$ ). Figure $2 \mathrm{~b}$ presents the proportion of patients who underwent coronary revascularization classified according to DWS (>0.34 vs. 0.34 or less). There was an absolute increase in the proportion of patients undergoing revascularization at a DWS value of 0.34 or lower.

\section{Discussion}

The novel finding of this study is that decreased DWS was associated with coronary revascularization in patients with stable angina. The important clinical implication of this finding is that lower DWS may be helpful to predict the need for coronary revascularization in patients with normal pre-procedural LVEF. This suggests a possible role of echocardiography in the diagnosis of stable angina. 


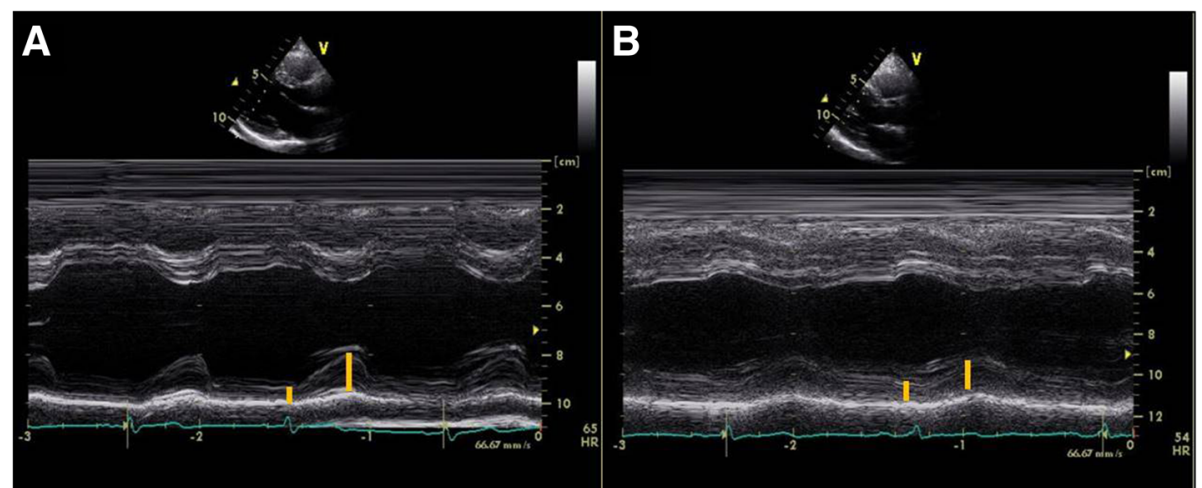

Fig. 1 Echocardiographic images of M-mode from parasternal long-axis view, a patient with normal diastolic wall strain (DWS, a) and a patient with decreased DWS (b)

Several conditions of either traumatic or atraumatic aetiologies can cause chest pain. To diagnose or exclude ACS, which is a life-threatening condition, prompt evaluation of chest pain using an algorithmic approach is important [2]. The most common causes of chest pain in outpatients are musculoskeletal and gastrointestinal causes; $10 \%$ have stable angina, $5 \%$ have respiratory conditions, and approximately 2 to $4 \%$ have acute myocardial ischaemia [14]. The target population of this study was the $10 \%$ of patients with stable angina. Among patients with suspected stable angina according clinical, laboratory, and imaging studies, 29\% (128/440) of patients needed coronary revascularization (percutaneous intervention: 113; bypass surgery: 14). However, stable angina could not be ruled out in all patients who did not

Table 3 Laboratory and other parameters

\begin{tabular}{llll}
\hline & Revascularization $(n=128)$ & No $(n=312)$ & $p$ \\
\hline Creatinine $(\mathrm{mg} / \mathrm{dl})$ & $0.99 \pm 1.15$ & $0.81 \pm 0.56$ & 0.029 \\
Total cholesterol & $167.4 \pm 42.7$ & $163.0 \pm 36.7$ & 0.301 \\
LDL & $100.0 \pm 36.2$ & $95.9 \pm 32.3$ & 0.278 \\
HDL & $42.2 \pm 10.8$ & $43.9 \pm 11.9$ & 0.187 \\
TG & $139.8 \pm 74.6$ & $125.2 \pm 75.6$ & 0.088 \\
hsCRP & $2.49 \pm 3.67$ & $2.04 \pm 3.11$ & 0.417 \\
BNP & $44.1 \pm 90.6$ & $32.4 \pm 52.8$ & 0.117 \\
CK-MB & $6.79 \pm 43.42$ & $1.74 \pm 2.17$ & 0.196 \\
Troponin I & $1.60 \pm 10.80$ & $0.11 \pm 0.54$ & 0.125 \\
Mean IMT (mm) & $0.77 \pm 0.24$ & $0.70 \pm 0.16$ & 0.009 \\
Carotid plaque (mm) & $80(90 \%)$ & $166(81 \%)$ & 0.125 \\
Max. plaque (mm) & $2.36 \pm 0.70$ & $2.02 \pm 0.57$ & $<0.001$ \\
ABI,rt & $1.15 \pm 0.12$ & $1.17 \pm 0.07$ & 0.176 \\
ABI,It & $1.13 \pm 0.11$ & $1.16 \pm 0.08$ & 0.103 \\
\hline Data are mean \pm stand & & &
\end{tabular}

Data are mean \pm standard deviation (SD) or or $\mathrm{n}(\%)$

$C H D$ coronary heart disease, $L A V I$ left atrial volume index, $L V$ EDD/ESD left ventricular end-diastolic and systolic dimension, $E F$ ejection fraction, $L V M I$ left ventricular mass index, DT deceleration time, RVSP estimated right ventricular systolic pressure undergo coronary revascularization. CAG was not an absolute indication in at least 312 study subjects. However, sometimes it is not feasible to perform non-invasive functional tests, such as the treadmill test or imaging tests (MPS with SPECT, stress echocardiography or MR perfusion) [3], in clinical practice. Hence, it is the standard practice at our centre to perform CAG if the suspicion of stable angina is high after clinical, laboratory and imaging studies (chest X-ray, ECG, or echocardiography). If pre-procedural echocardiography in patients with stable angina had some predictable parameters, it

Table 4 The angiography results of 127 patients who performed coronary revascularization

\begin{tabular}{ll}
\hline Characteristics & Number of patients (\%) \\
\hline 1 Vessel disease (VD) & $56(44 \%)$ \\
2 VD & $45(35 \%)$ \\
3 VD & $26(21 \%)$ \\
Number of stents & \\
$0^{\text {a }}$ & $19(15 \%)$ \\
1 & $76(60 \%)$ \\
2 & $26(20 \%)$ \\
3 & $5(4 \%)$ \\
4 & $1(1 \%)$ \\
Target lesion & \\
Involvement of left main & $9(7 \%)$ \\
Left anterior descending artery & $46(36 \%)$ \\
Left circumflex artery & $15(12 \%)$ \\
Right coronary artery & $24(20 \%)$ \\
Multiple & $33(26 \%)$ \\
Proximal & $50(39 \%)$ \\
Mid to distal & $44(35 \%)$ \\
Both (tandem lesion) & $33(26 \%)$ \\
\hline
\end{tabular}

${ }^{a}$ Of the 19 patients who did not undergo stent implantation, 14 patients underwent bypass surgery, 4 patients underwent balloon angioplasty, and 1 patient underwent thrombus suctioning 
Table 5 Uni-and multivariate analysis of the determinants of coronary revascularization in patients with stable angina

\begin{tabular}{llll}
\hline & Odds ratio & $95 \% \mathrm{Cl}$ & $p$ \\
\hline Univariate analysis & & & \\
Diastolic wall strain & 0.987 & $0.984-0.990$ & $<0.001$ \\
Left ventricular EF & 0.996 & $0.958-1.037$ & 0.856 \\
Left ventricular global strain & 0.888 & $0.805-0.979$ & 0.017 \\
E/E' & 1.111 & $1.046-1.179$ & 0.001 \\
Diastolic grading & 1.825 & $1.117-2.8983$ & 0.016 \\
Male gender & 2.076 & $1.344-3.207$ & 0.001 \\
Diabetes & 1.721 & $1.055-2.809$ & 0.030 \\
Current smoking & 1.624 & $1.025-2.574$ & 0.039 \\
Creatinine & 1.320 & $0.973-1.791$ & 0.075 \\
Mean carotid IMT & 7.687 & $1.851-31.926$ & 0.005 \\
Maximal plaque & 2.350 & $1.518-3.636$ & $<0.001$ \\
Multivariate analysis & & & \\
Diastolic wall strain & 0.875 & $0.806-0.950$ & 0.001 \\
Left ventricular global strain & 1.061 & $0.913-1.232$ & 0.440 \\
E/E' & 1.003 & $0.881-1.143$ & 0.960 \\
Diastolic grading & 1.656 & $0.720-3.811$ & 0.235 \\
Male gender & 1.211 & $0.512-2.864$ & 0.663 \\
Diabetes & 0.668 & $0.281-1.584$ & 0.359 \\
Mean carotid IMT & 13.041 & $1.277-124.827$ & 0.025 \\
Maximal plaque & 1.779 & $0.985-3.215$ & 0.073 \\
\hline
\end{tabular}

EF ejection fraction, IMT intima-medial thickness

would be helpful to determine the patients in whom CAG is indicated. Retrospectively, 312 (71\%) of our subjects did not require CAG. Non-invasive imaging plays a multifactorial role in patients with a known or suspected myocardial infarction. In particular, the advantages of echocardiography are to assess the cardiac structure and function and to detect RWMA (myocardial thickness, thickening and motion) [1]. Echocardiographic contrast agents can improve the visualization of the endocardial border and assess myocardial perfusion [15]. Tissue Doppler and strain imaging are very helpful tools for the quantification of global and regional function of LV $[11,15]$. However, these modalities have been primarily used in the setting of myocardial infarction. Therefore, we investigated echocardiographic parameters other than LV function or RWMA in the setting of stable angina.

It follows from the concept of the "ischaemic cascade" [16] that exercise or pharmacologic stress tests (ECG, echocardiography, nuclear test, etc.) are the major tests for evaluating coronary artery stenosis with a high accuracy and prognostic value in patients with clinical suspicion of myocardial ischaemia [17-20]. However, performing these tests before deciding to perform CAG is not always possible in clinical practice due to limitations of time and cost or due to contraindications. Considering the ischaemic cascade (the onset of ischaemia is followed by LV diastolic dysfunction, LV systolic dysfunction, ECG changes and angina, in that order) [16], we hypothesized that the evaluation of LV diastolic dysfunction might be helpful in patients with suspected stable angina. Fogelman et al. compared multiple posterior wall echograms taken before, during, immediately after, and $5 \mathrm{~min}$ after exercise in normal subjects vs. patients with angina; the angina patients showed not only a significantly decreased resting diastolic posterior wall velocity but also a remarkable slowing of the diastolic posterior wall velocity during angina [5]. DWS, a noninvasive, load-independent, and reproducible estimator of LV stiffness using 2D echocardiography, is used to assess wall distensibility in the absence of LV systolic
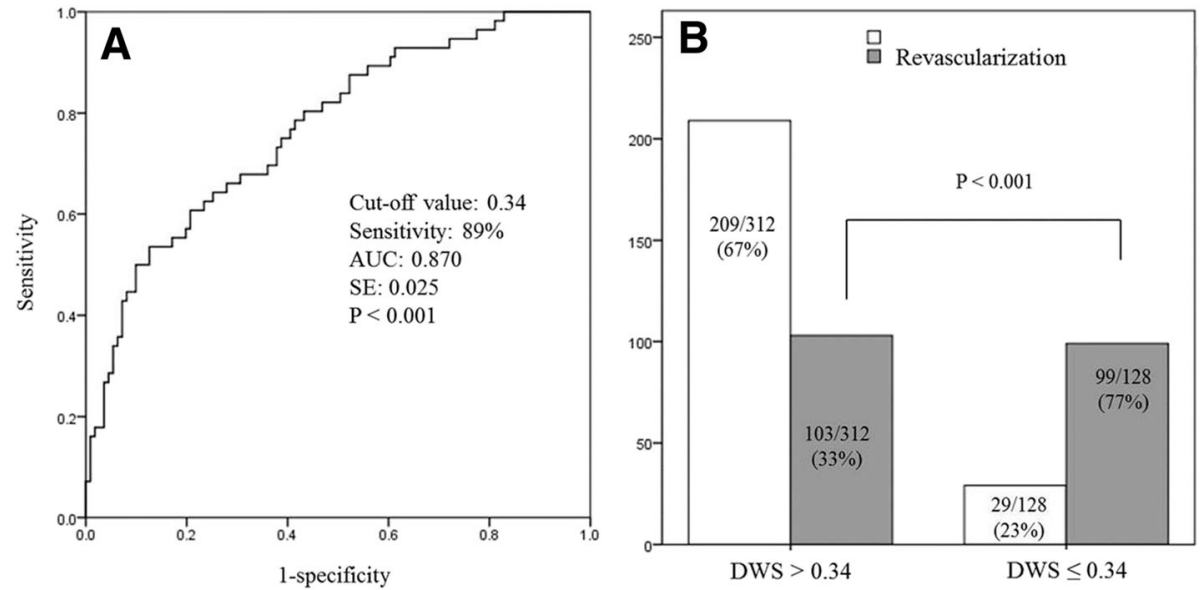

Fig. 2 a ROC curve showing the predictive value of DWS (less than 0.34 ) in coronary revascularization (sensitivity of $89 \%$ and specificity of $48 \%$, AUC: 0.870, SE: $0.025, p<0.001$, a). b Comparison of the proportion of patients who underwent coronary revascularization classified according to DWS (>0.34 vs. 0.34 or less) 
dysfunction [6]. Decreased DWS means increased diastolic myocardial stiffness and is associated with poor prognosis in patients with HFpEF [7] and HFrEF [8]. Hypertension can cause myocardial fibrosis in association with increased deposition of myocardial collagen, and myocardial fibrosis is responsible for myocardial stiffness. Even in patients with treated hypertension, decreased DWS (cut-off value $<0.34$ ) is associated with LV diastolic dysfunction [21]. The cut-off value of DWS for predicting coronary revascularization in our study was also 0.34 (sensitivity: $89 \%$ ). Takagi et al. reported that DWS is inversely correlated with post-exercise E/E' in elderly patients without obvious myocardial ischaemia, and DWS $\leq 0.33$ was defined as low DWS [22]. In addition, low DWS $(\leq 0.33)$ is associated with adverse LV remodelling (higher LV endsystolic volume and increased LV mass index) even in patients with normal LV diastolic function [9].

Atrial fibrillation (AF), the most common cardiac arrhythmia, is associated with the LV diastolic dysfunction [23]. Kang et al. observed that diastolic dysfunction (elevated E velocity, E/E') and dys-synchronous LA were associated with the occurrence of stroke in patients with paroxysmal AF, even in those with a similar CHAD (except prior stroke) score [24]. Uetake et al. reported that patients with AF had lower DWS than controls (0.35 vs. $0.41)$ and that decreased DWS $(<0.380)$ was a strong determinant of the prevalence of AF in patients with paroxysmal AF and structurally normal hearts [25].

Therefore, decreased DWS $\leq 0.33$ or 0.34 is definitely associated with increased LV stiffness and diastolic dysfunction in various diseases $[7-9,21,22,25]$ and is associated with poor prognosis in patients with $\mathrm{HFpEF}$, $\mathrm{HFrEF}[7,8]$ and $\mathrm{AF}[25]$.

\section{Limitations}

This study has a few limitations. First, few patients showed elevated pre-procedural cardiac biomarkers. Suspected ACS patients (typical chest pain, RWMA by echocardiography, ST elevation, or significant ST change in association with elevated cardiac biomarkers) were excluded from this study. Only a few patients who were very stable did not have resting chest pain and showed normal LVEF by echocardiography without discernible RWMA were included. Despite the insensitive and subjective nature of the echocardiographic evaluation of RWMA, the patients included in this study were not high-risk patients. Rarely, cardiac troponin can be elevated if the angina episode is prolonged in patients with chronic stable angina [26]. Second, we evaluated only epicardial coronary obstructions that needed revascularization. Therefore, microvascular angina or coronary spasm was not considered in this study. Third, we did not perform functional studies before CAG in all patients. Finally, the relatively small study population was a major limitation.

\section{Conclusions}

In summary, decreased DWS is associated with coronary revascularization in patients with stable angina. The important clinical implication of this finding is that lower DWS may be helpful to predict coronary revascularization in patients with normal pre-procedural LV EF. This implies a possible role of echocardiography in the diagnosis of stable angina.

\section{Abbreviations}

ACS: Acute coronary syndrome; CAD: Coronary artery disease; CAG: Coronary angiography; CB: Carotid bulb; CCA: Common carotid artery; CR: Complete revascularization; DWS: Diastolic wall strain; ECG: 12-lead-electrocardiography; EF: Ejection fraction; GS: Global strain; HFpEF: Heart failure with preserved LV ejection fraction; HFrEF: Heart failure with reduced LV ejection fraction; IMT: Intima-media thickness; IVSTd: End-diastolic interventricular septal thickness / PWTd: end-diastolic LV posterior wall thickness; LA: Left atrial; LV EDD: LV end-diastolic dimensions; LV: Left ventricular; LVMI: Left ventricular mass index; MPS with SPECT: Myocardial perfusion scintigraphy with single photon emission computed tomography; MR: Magnetic resonance; ROC: Receiver-operating-characteristic; RWMA: Regional wall motion abnormality; SD: Standard deviation; TDI: Tissue Doppler image;

TTE: Transthoracic echocardiography

\section{Acknowledgements}

There is nothing to declare with this study.

\section{Funding}

This research received no specific grant from any funding agency.

Availability of data and materials

The datasets used and/or analysed during the current study available from the corresponding author on reasonable request.

\section{Authors' contributions}

$J \mathrm{C}$ and MKK designed this study as the first author and corresponding author. $\mathrm{CH}, \mathrm{SMH}, \mathrm{SGJ}$ and HKK made the SPSS data together. KJC, SC, JRC and NL were involved in data acquisition and analysis in this study. All authors read and approved the final manuscript.

\section{Ethics approval and consent to participate}

This study complies with the Declaration of Helsinki and was approved by The Regional Committee of Kangnam Sacred Heart Hospital, Hallym University, Seoul, South Korea.

Consent for publication

Not applicable.

\section{Competing interests}

The authors declare that they have no competing interests.

\section{Publisher's Note}

Springer Nature remains neutral with regard to jurisdictional claims in published maps and institutional affiliations.

\section{Author details \\ ${ }^{1}$ Division of Cardiology, Hangang Sacred Heart Hospital, Hallym University Medical Center, Seoul, South Korea. ${ }^{2}$ Cardiology Division, Kangnam Sacred Heart Hospital, Hallym University Medical Center, Seoul, South Korea. ${ }^{3}$ Division of Cardiology, Department of Medicine, College of Medicine, Kangwon National University, Chuncheon, South Korea.}

Received: 3 August 2017 Accepted: 13 December 2017

Published online: 28 December 2017

\section{References}

1. Thygesen K, Alpert JS, Jaffe AS, Simoons ML, Chaitman BR, White HD. Joint ESC/ACCF/AHA/WHF task force for universal definition of myocardial 
infarction. 3rd universal definition of myocardial infarction. J Am Coll Cardiol. 2012;60:1581-98.

2. Skinner JS, Smeeth L, Kendall JM, Adams PC, Timmis A. Chest pain guideline development group. NICE guidance. Chest pain of recent onset: assessment and diagnosis of recent onset chest pain or discomfort of suspected cardiac origin. Heart. 2010:974-8.

3. Henderson RA, O'Flynn N. Guideline development group. Management of stable angina: summary of NICE guidance. Heart. 2012;98:500-7.

4. Genders TS, Steyerberg EW, Alkadhi H, Leschka S, Desbiolles L, Nieman K, et al. CAD Consortium. A clinical prediction rule for the diagnosis of coronary artery disease: validation, updating, and extension. Eur Heart J. 2011;32:1316-30.

5. Fogelman AM, Abbasi AS, Pearce ML, Kattus AA. Echocardiographic study of the abnormal motion of the posterior left Ventricular Wall during angina pectoris. Circulation. 1972;46:905-13.

6. Takeda Y, Sakata Y, Higashimori M, Mano T, Nishio M, Ohtani T, et al. Noninvasive assessment of wall distensibility with the evaluation of diastolic epicardial movement. J Card Fail. 2009:15:68-77.

7. Ohtani T, Mohammed SF, Yamamoto K, Dunlay SM, Weston SA, Sakata Y, et al. Diastolic stiffness as assessed by diastolic wall strain is associated with adverse remodelling and poor outcomes in heart failure with preserved ejection fraction. Eur Heart J. 2012;33:1742-9.

8. Soyama Y, Mano T, Goda A, Sugahara M, Masai K, Masuyama T. Prognostic value of diastolic wall strain in patients with chronic heart failure with reduced ejection fraction. Heart Vessel. 2017;32:68-75.

9. Kang MK, Ju S, Mun HS, Choi S, Cho JR, Lee N. Decreased diastolic wall strain is associated with adverse left ventricular remodeling even in patients with normal left ventricular diastolic function. J Echocardiogr. 2015;13:35-42.

10. Lang RM, Badano LP, Mor-Avi V, Afilalo J, Armstrong A, Ernande L, et al. Recommendations for cardiac chamber quantification by echocardiography in adults: an update from the American Society of Echocardiography and the European Association of Cardiovascular Imaging. J Am Soc Echocardiogr. 2015;28:1-39.

11. Dandel M, Lehmkuhl H, Knosalla C, Suramelashvili N, Hetzer R. Strain and strain rate imaging by echocardiography - basic concepts and clinical applicability. Curr Cardiol Rev. 2009;5:133-48.

12. Touboul PJ, Hennerici MG, Meairs S, Adams H, Amarenco P, Bornstein N, et al. Mannheim carotid intima-media thickness and plaque consensus (20042006-2011). An update on behalf of the advisory board of the 3rd, 4th and 5th watching the risk symposia, at the 13th, 15th and 20th European stroke conferences, Mannheim, Germany, 2004, Brussels, Belgium, 2006, and Hamburg, Germany, 2011. Cerebrovasc Dis. 2012;34:290-6.

13. Quadri G, D'Ascenzo F, Moretti C, D'Amico M, Raposeiras-Roubín S, Abu-Assi E, et al. Complete or incomplete coronary revascularisation in patients with myocardial infarction and multivessel disease: a propensity score analysis from the "real-life" BleeMACS (bleeding complications in a multicenter registry of patients discharged with diagnosis of acute coronary syndrome) registry. Eur Secur. 2017;13:407-14.

14. Ebell MH. Evaluation of chest pain in primary care patients. Am Fam Physician. 2011;83:603-5.

15. Flachskampf FA, Schmid M, Rost C, Achenbach S, deMaria AN, Daniel WG. Cardiac imaging after myocardial infarction. Eur Heart J. 2011;32:272-83.

16. Nesto RW, Kowalchuk GJ. The ischemic cascade: temporal sequence of hemodynamic, electrocardiographic and symptomatic expressions of ischemia. Am J Cardiol. 1987;59:23C-30C.

17. Schinkel AF, Bax JJ, Elhendy A, van Domburg RT, Valkema R, Vourvouri E, et al. Long-term prognostic value of dobutamine stress echocardiography compared with myocardial perfusion scanning in patients unable to perform exercise tests. Am J Med. 2004;117:1-9.

18. Mathias W Jr, Arruda A, Santos FC, Arruda AL, Mattos E, Osorio A, et al. Safety of dobutamine-atropine stress echocardiography: a prospective experience of 4,033 consecutive studies. J Am Soc Echocardiogr. 1999;12:785-91.

19. Gebker R, Jahnke C, Manka R, Hamdan A, Schnackenburg B, Fleck E, Paetsch I. Additional value of myocardial perfusion imaging during dobutamine stress magnetic resonance for the assessment of coronary artery disease. Circ Cardiovasc Imaging. 2008;1:122-30.

20. Ohman EM. CLINICAL PRACTICE. Chronic stable angina. N Engl J Med. 2016; 374:1167-76.

21. Liu YW, Lee WH, Lin CC, Huang YY, Lee WT, Lee CH, et al. Left ventricular diastolic wall strain and myocardial fibrosis in treated hypertension. Int J Cardiol. 2014;172:e304-6.
22. Takagi T, Takagi A, Yoshikawa J. Low diastolic wall strain is associated with raised post-exercise E/E' ratio in elderly patients without obvious myocardial ischemia. J Echocardiogr. 2014;12:106-11.

23. Tsang TS, Gersh BJ, Appleton CP, Tajik AJ, Barnes ME, Bailey KR, et al. Left ventricular diastolic dysfunction as a predictor of the first diagnosed nonvalvular atrial fibrillation in 840 elderly men and women. J AmColl Cardiol. 2002;40:1636-44

24. Kang MK, Han C, Chun K, Choi J, Choi S, Cho JR, Lee N. Factors associated with stroke in patients with paroxysmal atrial fibrillation beyond CHADS2 score. Cardiol. 2016;23:429-36.

25. Uetake S, Maruyama M, Yamamoto T, Kato K, Miyauchi Y, Seino Y, Shimizu $W$. Left ventricular stiffness estimated by diastolic wall strain is associated with paroxysmal atrial fibrillation in structurally normal hearts. Clin Cardiol. 2016;39:728-32.

26. Thygesen K, Mair J, Katus H, Plebani M, Venge P, Collinson P, et al. study group on biomarkers in cardiology of the ESC working group on acute cardiac care. Recommendations for the use of cardiac troponin measurement in acute cardiac care. Eur Heart J. 2010;31:2197-204.

\section{Submit your next manuscript to BioMed Central and we will help you at every step:}

- We accept pre-submission inquiries

- Our selector tool helps you to find the most relevant journal

- We provide round the clock customer support

- Convenient online submission

- Thorough peer review

- Inclusion in PubMed and all major indexing services

- Maximum visibility for your research

Submit your manuscript at www.biomedcentral.com/submit
Biomed Central 